\title{
Molecular genetics and epidemiology of epithelial ovarian cancer (Review)
}

\author{
SHOJI HARUTA, NAOTO FURUKAWA, YORIKO YOSHIZAWA, TAIHEI TSUNEMI, AKIRA NAGAI, \\ RYUJI KAWAGUCHI, YASUHITO TANASE, SHOZO YOSHIDA and HIROSHI KOBAYASHI
}

\author{
Department of Obstetrics and Gynecology, Nara Medical University, Nara 634-8522, Japan
}

Received July 10, 2011; Accepted August 19, 2011

DOI: 10.3892/or.2011.1456

\begin{abstract}
This review summarizes data from recent molecular genetic and epidemiology studies of the generic term 'female pelvic cancer'. The English-language literature was reviewed for genetic, epigenetic, epidemiologic and environmental risk factors. There are well-documented disparities among racial and ethnic groups with respect to epithelial ovarian cancer (EOC) prevalence. In the case of the serous histological subtype, primary EOC, fallopian tube cancer and peritoneal cancer could be regarded as a single disease entity. However, EOC is not a single disease. Comparing the profile of EOC between Japanese and Caucasians, clear cell carcinomas $(27.6 \%)$ are more common in Japan, possibly with fewer serous adenocarcinomas $(40.7 \%)$. This may reflect a proportional increase. The Japanese may exhibit a higher proportion of malignant transformation of endometriosis compared to the United States population. Although some part of the molecular genetic pathogenesis has been unveiled, the complete events of molecular genetic epidemiological changes associated with EOC remain to be identified. This review focuses on current knowledge of the genetic and environmental factors affecting the development of EOC, and outlines future challenges in its pathogenesis research.
\end{abstract}

\section{Contents}

1. Introduction

2. Search strategy and selection criteria

3. Epidemiology of female pelvic cancer

4. Epidemiology of epithelial ovarian cancer

5. Pathogenesis

6. Risk factors

Correspondence to: Dr Hiroshi Kobayashi, Department of Obstetrics and Gynecology, Nara Medical University, 840 Shijo-cho, Kashihara, Nara 634-8522, Japan

E-mail: hirokoba@naramed-u.ac.jp

Key words: epidemiology, epithelial ovarian cancer, genetics, pathogenesis
7. EOC candidate genes and susceptibility genes

8. Future research

\section{Introduction}

Human cancer results from multiple environmental, epigenetic and genetic events over time. The etiology of epithelial ovarian cancer (EOC) is not well understood but is likely to involve both genetic and environmental factors. Epidemiological evidence suggests that disparities may exist in the pathological characteristics of EOC among different ethnic groups. First, age-adjusted incidence rates for EOC are higher among Whites compared to the Japanese. Second, in Europe and North America, approximately two-thirds of cases of EOC are of high-grade serous adenocarcinoma (SAC) type, which is a rapidly growing highly aggressive tumor (1). Third, clear cell carcinoma (CCC) of the ovary is more common in Japan, possibly with fewer SAC cases. CCC is a slow-growing tumor and has been associated with endometriosis (1). Finally, there are well-documented disparities among genetic mutations, chromosome instability, gene methylation, and activation of oncogenes or inhibition of tumor suppressor genes with respect to EOC pathogenesis. Thus, EOC is not a single disease entity (2).

Although recent genetic studies have revealed gene abnormalities which may be associated with the risk of EOC, the development of this cancer is also influenced by environmental factors. This review focuses on the current knowledge of the genetic and environmental factors affecting the development of EOC, and outlines future challenges in its pathogenesis research.

\section{Search strategy and selection criteria}

A computerized literature search was performed to identify relevant studies reported in the English language. We searched the electronic databases PubMed Medline (http:// www.ncbi.nlm.nih.gov/sites/entrez) from the date of database development to May 2011. All abstracts were reviewed by two investigators to identify studies for full-text review. Discrepancies were resolved by consensus. The medical subject heading terms used included cancer, carcinoma, fallopian tube, peritoneal, genetic, epigenetic, environmental, 
epidemiology, risk factor, incidence, pathogenesis, etiology, germline, somatic, mutation, susceptibility gene, single nucleotide polymorphisms (SNPs), racial, ethnic, or histology with epithelial ovarian cancer. We review the literature for EOC candidate gene studies and common germline/somatic genetic variation based on genome-wide gene expression profiling studies and SNP approach. Furthermore, the progressive knowledge on predisposing environmental conditions has been review by molecular epidemiological studies. We also review the reference lists of identified articles and relevant review articles on the subject to identify studies that may have been missed in the initial database search. Initially, 106 potentially relevant studies were identified by screening electronic databases, and 35 peer-reviewed journal articles were additionally identified from references in each article.

Data source in Japan. The newly diagnosed cancer cases at all ages in the gynecologic cancer registration database were used in this analysis. Gynecologic cancer cases which are diagnosed and treated at the major hospitals in Japan have been registered to the Japan Society of Obstetrics and Gynecology (JSOG), the main registration office (http://www. jsog.or.jp). Every case of cancer was identified and added to the pool of cases. Data on all registered cases were re-fed on an excel programme and carefully checked for repetition and apparent errors. The verified cases were used to present an estimate of the incidence of cancer. For this analysis 5,277 EOC cases diagnosed and registered in 2009 in JSOG were considered. These data represent about $80 \%$ of the major hospitals (464/580) in Japan.

\section{Epidemiology of female pelvic cancer}

The generic term 'female pelvic cancer' contains primary ovarian cancer, fallopian tube cancer and peritoneal cancer The age-adjusted incidence rates for ovarian cancer (11.2 per 100,000 women per year) was higher than for peritoneal (0.68 per 100,000 women per year) or fallopian tube $(0.37$ per 100,000 women per year) cancer in the United States during the period 1995-2004 (3). Fallopian tube and peritoneal cancers are uncommon malignancies with similarities to EOC, including histological, clinical, and etiological factors (3).

Fallopian tube cancer has been defined morphologically by the invasion of the peritoneal surfaces with minimal or no involvement of the ovaries (4). Recent genetic and immunohistochemical studies strongly suggest that high-grade SAC involving the ovary likely arises from distal fallopian tube epithelium, indicating that some ovarian cancers develop in the fallopian tube and are then spread to the ovary $(1,2,5)$. In other words, less than half of ovarian cancer cases that are diagnosed as early stage may arise in the ovary, whereas the remaining more than half that are diagnosed as advanced stage may arise in fimbrial epithelial cells.

Carlson et al reported that the fimbria may also be the source of primary peritoneal cancer, suggesting that SAC may originate in the tubal mucosa but grows preferentially at a remote peritoneal site (6). Another hypothesis is that endometrial intraepithelial carcinoma cells with increased anti-apoptosis expelled during menstruation or retrograde hemorrhage may survive in ectopic locations possibly via a mechanism as is accepted for the implantation theory of endometriosis, resulting in the development of peritoneal cancer. In view of these findings, fallopian tube cells or uterine endometrial intraepithelial carcinoma cells is a precursor lesion of SAC, originating in the tubal epithelial cells or uterine endometrial cells and spreading into the intraperitoneal cavity (7). Taken together, a substantial number of female pelvic cancer cases appear to primarily develop in the fallopian tube and peritoneum and secondarily involve the ovary.

Time trend analyses exhibited a rise in the rates of peritoneal and fallopian tube cancers. Rates for fallopian tube cancer peaked among women 70-74 years of age. Interestingly, the incidence of fallopian tube cancer specifically increased by $\sim 80 \%$ (3). One possibility is that the shift in registration coding was denoted for ovary and fallopian tube cases, possibly due to the gained popularity of the new theory that a subset of ovarian cancer arises from fallopian tube epithelial cells (3). There is no doubt that EOC had been over-diagnosed at the expense of fallopian tube and peritoneal cancers.

Based on ethnicity, there were few cases of fallopian tube cancers among Asian-Pacific Islanders. More than 90\% of fallopian tube cancers are SAC which are histologically indistinguishable from SAC of the ovary $(8,9)$. Endometrioid adenocarcinomas (EAC) were the next most commonly specified fallopian tube histological subtype. CCC and mucinous adenocarcinoma (MAC) were rarely diagnosed in the fallopian tube.

Peritoneal cancer is diagnosed at a more advanced age (67 years) than fallopian tube cancer (64 years) and EOC (63 years). Non-SAC are extremely uncommon in the peritoneal cancer. This pattern was consistent for all racial and ethnic groups, although Black, Asian-Pacific Islander, and Hispanic women tended to be diagnosed at younger ages compared to White women. Both fallopian tube and peritoneal cancers are rare so etiologic studies are difficult to conduct, requiring large collaborations among research centers.

\section{Epidemiology of epithelial ovarian cancer}

Despite recent diagnostic and therapeutic advances, EOC still carries a poor prognosis. More than 200,000 new cases of EOC in the world are registered at the International Cancer Study Agency every year and >120,000 women die from EOC. EOC has an age-adjusted incidence of 12.8, 8.8, 7.7 and 7.6 per 100,000 women per year in the UK, the United States, France and Japan, respectively $(20,52)$. The Surveillance, Epidemiology and End Results (SEER) Program is a premier source for cancer statistics in the United States (http://seer. cancer.gov/). EOC is the leading cause of death from gynecological malignancy in the developed world, accounting for $4 \%$ of the deaths from cancer in women. It is the fifth most common cause of cancer death in women trailing behind lung, breast, colorectal, and pancreatic cancers (10).

The estimated average number of EOC cases diagnosed each year in the United States between 1979 and 2000 is 25,580 (11). The average number of EOC-related deaths expected to occur in the United States during this period was 16,090 (11). It was estimated that 21,880 women would be diagnosed with ovarian cancer and 13,850 women would die of cancer of the ovary in 2010. 
About 2,300 new cases of EOC are found in women in Canada each year (12). EOC affects about 1 in 70 Canadian women. About 1,600 Canadian women die each year of this disease, making it the fifth ranking cause of cancer-related deaths. EOC is a major cause of morbidity and mortality in Western countries.

About 8,300 new cases of EOC are found in women in Japan each year (http://ganjoho.ncc.go.jp/public/index.html). About 4,600 Japanese women die each year of this disease. Overall, the latest population-based cancer registry provides that the incidence rate of EOC was 9.09, 7.28, and 6.49 per 100,000 population for the United States, Canada, and Japan, respectively.

The incidence rate for EOC remains controversial. Goodman and Shvetsov showed that the time trend analyses between 1973 and 2005 in the United States exhibited a decline by $27 \%$ in EOC incidence (3). Oral contraceptive (OC) use reduces the risk of ovarian cancer and endometrial cancer, while an increased incidence of breast cancer has occurred. OC usage can also diminish the incidence of EOC, in both the general population, as well as in patients with BRCA1/2 germline mutations (13). OC use may be protective (14). In northern Europe and North America, EOC rates have remained almost constant over the last 2-3 decades (15). By contrast, a ten-year cohort study in Canada demonstrated that the number of cases of EOC constantly increased between 1997 and 2006 (12). An increase in EOC rates has also been reported in Japan (15). Although the reasons for the higher ovarian cancer incidence rates in Japan are unknown, the trends may be due to changes in risk factors, such as diet and environmental factors. Further studies should confirm if this change is a geographically widespread or a localized phenomenon.

\section{Pathogenesis}

The ovarian surface epithelium (OSE) and the peritoneum share a common embryonal heritage, deriving from coelomic epithelium (16). Similar to the ovary, the fallopian tube is derived from the Müllerian ducts (17). The fimbria, peritoneum and OSE are parts of the common origin and may express common features. SAC is the most commonly diagnosed histological type for all anatomic sites of female pelvic cancer. Clinically, patients diagnosed with fallopian tube cancer and primary peritoneal cancer are treated using the same therapeutic strategies including the same surgical and chemotherapeutic approaches as those for EOC, because of the similarities in their biological and clinical behavior $(4,18)$. Cytotoxic chemotherapy (taxane and platinum) followed by debulking surgery has an important role in the treatment of these diseases. The biological, pathological and clinical similarities between fallopian tube cancer, peritoneal cancer and EOC support the likelihood of a common molecular pathogenesis (19).

EOC is not a single disease entity and has been classified into four major histopathological subtypes. Approximately two-thirds of cases of EOC worldwide are of the high-grade $\mathrm{SAC}$ type. The remaining cases are comprised of different tumor types (e.g., EAC, CCC and MAC). SAC, EAC/CCC, and MAC tumors resemble the phenotypes of the fallopian tube, proliferative endometrium/gestational endometrium, and endocervix/gastrointestinal tract, respectively $(9,19)$.
It was reported in the past that the EOC arises in the OSE or cortical inclusion cysts formed in the cortical stroma after cyclic ovulation, which was explained by coelomic metaplasia from the peritoneum (20). A growing body of evidence has accumulated that the distal fallopian tube epithelial cells, but not the OSE or inclusion cyst, may be the source of highgrade SAC (21). Multiple genetic and epigenetic changes are involved in the molecular pathogenesis of SAC, for example, germline/somatic mutations in the p53 tumor suppressor gene (TP53). TP53 is essential for the preservation of genome integrity. The p53 protein functions to induce growth arrest, DNA repair, senescence, and apoptosis in response to cellular stress, including DNA damage. Somatic mutations in TP53 are present in $>90 \%$ of sporadic SAC. Thus, mutant TP53 is considered to be a driver mutation in the pathogenesis of SAC.

However, loss of TP53 function must be accompanied by at least one more genotoxic event (including BRCA1/2 functional inactivation) to produce the malignant phenotype. This is in keeping with a model of carcinogenesis, in which independent risk factors operate at multiple points in the serous carcinogenic spectrum. Oncogenic mutations of specific genes probably represent a secondary hit in a multistep process.

For example, oncogenes with predominant roles in lung cancer include not only TP53 but also EGFR (epidermal growth factor receptor), MYC (v-myc myelocytomatosis viral oncogene homolog), KRAS (v-Ki-ras2 Kirsten rat sarcoma viral oncogene homolog), PIK3CA (phosphoinositide-3-kinase, catalytic, $\alpha$ polypeptide), NKX2-1 (NK2 homeobox 1), ALK (anaplastic lymphoma receptor tyrosine kinase), RB1 (retinoblastoma 1), CDKN2 (cyclin-dependent kinase inhibitor 2A) or others (22). The risk for breast cancer is caused by mutations in TP53 and others, including BRCA1/2, STK11 (serine/ threonine kinase 11), PTEN (phosphatase and tensin homolog), CDH1 (cadherin 1, type 1, E-cadherin), NF1 (neurofibromin 1) or NBN (nibrin) (23). Furthermore, about 6 or 7 mutations are required for colorectal cancer to develop, which include TP53, KRAS, APC (adenomatous polyposis coli) and others. Similar to other cancers, the second-hit may aggravate gene mutation in the TP53-mutated cells, leading to the generation of SAC. These findings allow us to speculate that the second hits including organ-specific, environmental or ethnic factordependent gene aberration can produce SAC when there is a mutation to a single allele of a TP53 gene. The molecular genetics of each histological type are described in the 'EOC candidate genes and susceptibility genes' section.

\section{Risk factors}

Collection of epigenetic, genetic, environmental, social and psychological data is necessary to assess their interaction. A number of epidemiologic studies have evaluated a variety of risk factors for EOC $(10,24)$. There are well-documented disparities among racial and ethnic groups with respect to EOC prevalence, age at diagnosis, pathological and clinical features, such as stage, histology, chemosensitivity, clinical complications and mortality. As shown in Table I, several risk factors have been associated with EOC.

Age. Although EOC may occur at any age, it is more common in patients $>50$ years of age (10). The incidence of EOC rose 
Table I. Risk factors for EOC.

Increased risk

Decreased risk

Age, older

Early menarche

Late menopause

Low parity

Delayed childbearing

Estrogen replacement therapy for more than five years

High-fat diet

Ethnicity, White

Family history suggesting genetic predisposition

Genetic syndromes

Endometriosis

Talc use/asbestos exposure

Chronic inflammation
Age, younger

Late menarche

Early menopause

Multiparity

Breastfeeding for 18 months or more

Oral contraceptive use

Low-fat diet

Ethnicity, Japanese

This study reports on a literature survey of epidemiological studies on EOC risks.

monotonically with increasing age up to the age of 75-79 years, before declining slightly among women 80 years. Everpregnant women had a lower risk of EAC or CCC than did nulliparous women (19).

Diet. Fat diet intake rates differ by place of birth, acculturation and socioeconomic status. Dietary components related to high socioeconomic status, animal fat and red meat, and physical inactivity, have been associated with $\operatorname{EOC}(4,18)$. A low-fat dietary pattern reduces the incidence of EOC among postmenopausal women (25). Indeed, dietary constituents peculiar to a typical Western diet affect ovarian carcinogenesis in various ethnic minority groups including the Japanese. Fat and red meat diet intake rates increased in the 1990s in Japanese adolescents. To what extent this factor plays a role in the etiology of EOC in Japan remains to be fully elucidated.

Hormone therapy. There may be a small increased risk of EOC associated with longer-term use of hormone therapy. Although progestin may mitigate some of the detrimental effects of estrogen, estrogen therapy (ET) as well as estrogen/progestin therapy (EPT) are risk factors for EOC (26). Regardless of the duration of use, the formulation, estrogen dose, regimen, progestin type, and route of administration, hormone therapy was associated with an increased risk of EOC (27).

There appears to be no differential impact of any therapy on histological subtypes (26). However, some reports showed that ever-use of non-contraceptive hormones or estrogens is associated with increased risk of CCC (19). Furthermore, post-menopausal ERT may be a risk factor associated with both CCC and EAC (28). EACs are related to pregnancy and tubal ligation, while CCCs are the only type associated with non-contraceptive hormone use (19). It is uncertain whether this association is correlated with the absence of estrogen receptor- $\alpha$ and progesterone receptor expression among most
CCC types $(19,29,30)$. In addition, the risk associated with ERT is much larger in women with an intact genital tract than in those with a history of either hysterectomy or tubal ligation (28).

Inflammation and endometriosis. Inflammation entails oxidative stress, cell and DNA damage, and elevations of proinflammatory cytokines and prostaglandins, all of which may be mutagenic. Some cancers have been estimated to be attributable to underlying inflammation, including colon, prostate, liver, pancreatic and cervical cancers. Factors contributing to chronic inflammation appear to be associated with an increased risk of EOC (31). Inflammation-mediated EOC represents an example of a possible gene-environment interaction. For example, chronic inflammation caused by talc and/or asbestos is a known risk factor for EOC and peritoneal cancer (24). There is accumulating evidence suggesting that as aspirin use increased, EOC risk decreased (24). NSAIDs may also decrease EOC risk.

Hysterectomy acts as a protective factor, possibly by diminishing the likelihood that the fallopian tube epithelium, OSE and peritoneum will be exposed to environmental initiators of inflammation $(18,19)$. Tubal ligation can also block passage of inflammatory agents from the genital tract to the pelvic cavity $(18,19)$. Tubal ligation is associated with a reduced risk of EAC and CCC, but not MAC $(4,19)$.

Furthermore, there are well-defined risk factors including a history of endometriosis, which are known to predispose susceptible individuals to developing this disease. A prospective study in Japan directly showed that, during a follow-up of up to 17 years of an ovarian endometrioma cohort $(n=6,398)$, 46 incidences of EOC were identified, showing that the EOC risk was significantly elevated in patients with ovarian endometrioma [standardized incidence ratio $(\mathrm{SIR})=8.95](32,33)$. Endometriosis morbidity likely contributes to this disparity 


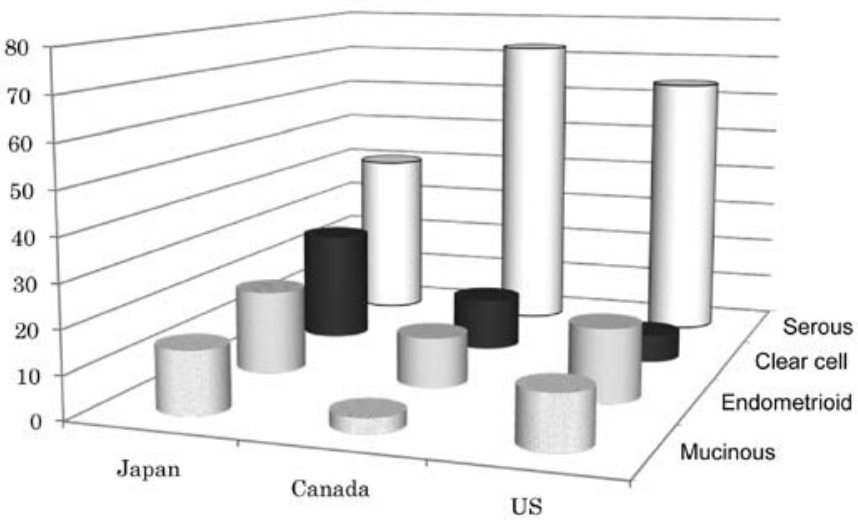

Figure 1. Race- and histological subtype-specific incidence rates of EOC. Plots are the race-specific incidence rates of EOC by histological subtype.

but does not seem to fully account for it. Retrograde menstruation or ovarian hemorrhage carries highly pro-oxidant factors, such as heme and iron, into the peritoneal cavity or ovarian endometrioma, contributing to the underlying inflammation. A histologically normal ectopic endometrium bears genetic damages caused by iron-dependent persistent oxidative stress via a Fenton chemical reaction (34-36). Subclinical endometriosis may predispose women to endometriosis-associated ovarian cancer (EAOC) due to their continued exposure of free iron. Epidemiological studies support a close link between iron overload and carcinogenesis (37). Pathological conditions such as hemochromatosis, renal cancers, chronic viral hepatitis $\mathrm{B}$ and $\mathrm{C}$, exposure to asbestos fibers and endometriosis have been recognized as iron overload-associated risks for human cancer (38).

Race. Racial and ethnic disparities exist in the incidence, histological subtype, mortality and clinical trends of EOC. Traditional socioeconomic and behavioral risk factors cannot fully explain racial disparity in EOC. This unexplained gap has led to speculation that genetic factors contribute to racial disparity in EOC. The disparities have been demonstrated for not only differences in gene or SNP frequency, but also differences in environmental exposures or interaction between multiple genes and environment. Thus, genetic predisposition may account for some of the racial differences in the histological patterns of EOC (19).

White women had the highest rates for all three female pelvic malignancies. The most disparate incidence rates were for cancer incidence among Black women compared to White women, which were 0.62 for ovary, 0.39 for peritoneum, and 0.72 for fallopian tube. The age-adjusted incidence rate of EOC was 12.8 per 100,000 women per year in the United States; White 13.5, American Indian/Alaskan Native 10.6, Hispanic 10.6, Black 10.0, and Asian/Pacific Islander 9.9 per 100,000 women per year (http://seer.cancer.gov/). Compared with the residents of Western Europe, North America and Australia, those in Japan have a lower incidence of EOC (39). The incidence in native Japanese is lower than in JapaneseAmericans, who themselves have rates similar to Americans. This geographical variation in the incidence of the disease, and the pattern of increased incidence in immigrants from low- to high-risk areas, suggests a major contribution from environmental factors.

One study reported that, although the incidence of EOC is much lower in Oriental than Caucasian women, the proportion of different histological types, stage at presentation and survival from EOC in Oriental women does not differ from that in Caucasians (40). Some studies comparing the profile of EOC between Japanese and Caucasians showed, however, that the two groups were significantly different in the pathology of EOC, with a greater propensity for the CCC among the Japanese. Fig. 1 shows that disparities may exist in the pathological characteristics of EOC among different ethnic groups. Alternatively, additional concerns in the interpretation of the data include possible differences in the classification of cancers by the pathologists in the various hospitals.

Histology. Using multivariate analysis, age, stage, grade, histology, treatment strategy, and residual tumors were identified as independent predictors of survival (41). There are biological differences in the behavior of the major tumor types (10). The distribution of EOC types differs in patients with low-stage vs. high-stage EOC. High-grade SACs account for $35.5 \%$ of stage I/II tumors and $87.7 \%$ of stage III/IV tumors (42). In contrast, CCCs (26.2 vs. $4.5 \%$ ), EACs (26.6 vs. $2.5 \%$ ), and MACs (7.5 vs. $1.2 \%$ ) were more common among the lowstage vs. high-stage tumors (42). CCC are more likely to be diagnosed at early-stage. The 5-year disease free survival of patients with CCC is worse compared to SAC: 85.3 vs. $86.4 \%$ for stage I, 60.3 vs. $66.4 \%$ stage II, 31.5 vs. $35.0 \%$ stage III, and 17.5 vs. $22.2 \%$ for stage IV, respectively (41). Adjusted for stage, women with $\mathrm{CCC}$ have a poorer prognosis compared to SAC. Retroperitoneal lymph node metastases were found in $13.6 \%$ of SAC, $7.9 \%$ CCC, $7.3 \% \mathrm{EAC}$, and $3.8 \%$ of MAC (40). Women with CCC present at a younger age. They are more likely to be Asian.

In general, approximately two-thirds of EOC cases in Europe and America are of the high-grade SAC type. Data from the British Columbia Cancer Agency $(n=2,555)$ demonstrated that the overall frequency of tumor types was as follows: $68.1 \%$ high-grade SAC, $12.2 \% \mathrm{CCC}, 11.3 \% \mathrm{EAC}$, $3.4 \%$ MAC, and 3.4\% low-grade SAC (42) (Fig. 1). This distribution was found to be very similar in cases $(n=410)$ from the Washington Hospital Center (42). Of women with EOC in the United States, $49.3 \%$ SAC, $13 \%$ EAC, 9.7\% MAC, 5\% had CCC , and 23\% had unspecified histologies (41) (Fig. 1). Studies published in Finland (43) and Denmark (10) in the past several decades suggest rates comparable to the United States. Among white women, the reported incidence rates for invasive SAC, EAC, and CCC increased during 1978-1998 (44). Hispanic and Black women had low rates of EAC and CCC tumors (39). The proportion of CCC histology was significantly higher in Asians vs. Whites, Blacks, and others (11.1 vs. 4.8, 3.1, and 5.5\%) (41). Asian women were more commonly diagnosed with CCC than SAC when compared with Caucasian women $(8,16,19)$. Among Asian women, Japanese women had an increased incidence of CCC (Fig. 1). These differences may be due primarily to a lower rate of SAC or a higher rate of CCC in Japanese women.

The median age of the patients was 64 years; with CCC patients presenting at younger age (55 years) in the United States (41). We examined age-specific incidence rates of 


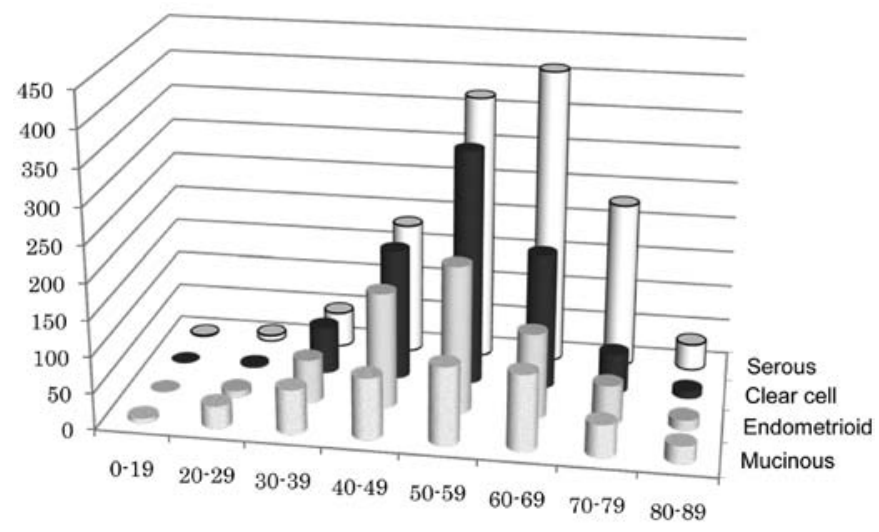

Figure 2. Age distribution of EOC by histological subtype in Japan. Plots are the age distribution of EOC Japanese patients. The horizontal axis represents the grouping of age at diagnosis. The vertical axis represents the number of patients in each age group for that histological subtype [serous, mucinous, endometrioid adenocarcinoma and clear cell carcinoma (SAC, MAC, EAC and $\mathrm{CCC})]$.

histological subtypes of EOC in Japan. Three thousand four hundred and twenty new EOC cases registered in the JSOG registry were considered for analysis. Among EOC, SAC are the most frequent subtype (40.7\%), followed by CCC (27.6\%) (Fig. 1). Histological subtypes of EOC according to age are presented in Fig. 2. The median age of EOC diagnosed in Japan was 49 years of age. There were $42 \%$ of EOC diagnosed in the 50-69 age group. SAC were most common in the 60-69 age groups. Other epithelial tumors were most common in the 50-59 age groups.

There are several possibilities to explain the fact that SAC are less common in Japanese. First, in Japan, a positive rate of alteration in TP53 may be lower than that (>90\%) of p53 in Caucasians. Second, some specific SNPs in TP53 or its related genes may be associated with increased odds of somatic mutation in SAC in Caucasians, but not in Japanese. Third, it may be due to the epigenetic or genetic heterogeneity of 'post-TP53' mutations. One should investigate the association of TP53 mutations or SNPs with SAC in Japanese women with a lower incidence of SAC. Finally, proteins that bind and inactivate p53 protein such as MDM2 (Mdm2 p53 binding protein homolog), overexpressed in a variety of human cancers, may be down-regulated in SAC in Japanese women.

The precise incidence of CCC is unknown, but it is generally reported to be $3.7-12.1 \%$ of all histological subtypes among EOC worldwide (45). CCC occurs more frequently in Japan than in Western countries (16). The most common histology in Japan was SAC (40.7\%) followed by CCC (27.6\%) and EAC (18.3\%), as in previous reports in Taiwan and Italy (46-49). CCC are more common in Japan, possibly with fewer SAC. This may reflect a proportional increase.

$\mathrm{CCC}$ and EAC are closely associated with endometriosis, suggesting that the precursor appears to be endometriosis $(32,46,50)$. Indeed, the prevalence of endometriosis in EOC has been calculated to be $4.5,1.4,35.9$, and $19.0 \%$ for SAC, $\mathrm{MAC}, \mathrm{CCC}$ and EAC, respectively. Women with endometriosis were at increased risk for developing CCC followed by EAC. Although some endometriosis lesions may predispose to $\mathrm{CCC}$ and EAC, both of these cancers differ from the other histological types with respect to their clinical characteristics and carcinogenesis $(32,33,49)$. Endometriosis is a complex genetic trait which affects up to $10 \%$ of women in their reproductive years (51). It causes severe dysmenorrhea, pelvic pain and subfertility (52). It is known that prevalence of endometriosis is probably an underestimation since an unknown proportion of women suffering of endometriosis do not consult for their symptoms. Interestingly, some, but not all, studies have found a higher prevalence of endometriosis among Asian women (53). Furthermore, although the risk of malignant transformation of endometriosis appears to be very limited $(\sim 1 \%)$, Japanese may have an increased susceptibility to cancer. During follow-up of the ovarian endometrioma cohort in Japan, the EOC risk was elevated significantly among patients with ovarian endometrioma ( $\mathrm{SIR}=8.95,95 \% \mathrm{CI}=4.12-15.3$ ) (32). Women with endometriosis in the United States had an elevated risk ( $\mathrm{SIR}=2.48$; 95\% $\mathrm{CI}=1.3-4.2$ ), with a further elevated risk among those with primary infertility ( $\mathrm{SIR}=4.19,95 \% \mathrm{CI}=2.0-7.7)(54)$. These data allow us to speculate that Japanese may exhibit a higher proportion of malignant transformation compared to the United States population.

CCCs are more closely related to type I tumors (55). CCCs develop along two pathways; CCC with an adenofibromatous background and those without an adenofibromatous background (55). Endometriosis was found in both types of tumors, but tumors arising in endometrioma were more frequent with tumors without an adenofibromatous background (55).

One of the major clinical problems in the clinical management of CCC is its poor sensitivity to first-line platinum-based chemotherapy and the association with a worse prognosis than the more common SAC. Patients with CCC, especially in advanced stage or recurrent disease, have a worse progression free survival and overall survival when compared with patients with a SAC histology (14). Recent biochemical studies based on genome-wide expression analysis technology have noted specific expression of a transcription factor, hepatocyte nuclear factor-1 $\beta$ (HNF-1 $\beta$ ) in CCC (56). Up-regulation of HNF-1 $\beta$ expression may lead to a highly chemoresistant nature resulting in a poor prognosis (unpublished data). The HNF-1 $\beta$-dependent pathway of CCC provids new insight into the regulation of apoptosis, glycogen synthesis and resistance of CCC to anticancer agents (57).

\section{EOC candidate genes and susceptibility genes}

EOC is a complex disease that may result from the interaction of genetic and environmental factors. The number of genetic factors known to contribute to EOC is large and growing. There are well-defined risk factors including genetic syndromes (e.g., familial ovarian cancer syndrome), which are known to predispose susceptible individuals to developing this disease. Genetic mutation, chromosome instability, gene methylation, and activation of oncogenes or inhibition of tumor suppressor genes have been found to disrupt the expression profiles of some important proteins or enzymes in various cellular signaling pathways. Environmental factors can modify the disease risk, which is associated with a given genetic risk factor.

We initially reviewed candidate genes and common genetic variations. High-grade SACs are chromosomally unstable 
Table II. Moderate low-penetrance susceptibility genes for EOC.

\begin{tabular}{|c|c|c|c|}
\hline Functions & Official symbol & Name & Ref. \\
\hline \multirow[t]{2}{*}{ Hormone receptors } & ESR1 & Estrogen receptor 1 & 66 \\
\hline & PGR rs1042838 & Progesterone receptor & 70 \\
\hline \multirow[t]{7}{*}{ Cell cycle regulators } & CCND1 & Cyclin D1 & 71 \\
\hline & CDKN2A & Cyclin-dependent kinase inhibitor 2A (melanoma, p16, inhibits CDK4) & 71 \\
\hline & CDKN1B & Cyclin-dependent kinase inhibitor 1B (p27, Kip1) & 71 \\
\hline & CDK6 & Cyclin-dependent kinase 6 & 71 \\
\hline & MDM2 & Mdm2 p53 binding protein homolog & 71 \\
\hline & $\mathrm{XRCC} 2$ & X-ray repair complementing defective repair & 71 \\
\hline & BABAM1 & BRISC and BRCA1 A complex member 1 & 72 \\
\hline \multirow[t]{2}{*}{ Cytokines } & IL-1RA & Interleukin-1 receptor antagonist & 73 \\
\hline & IL-18 rs1834481 & Interleukin 18 (interferon- $\gamma$-inducing factor) & 73 \\
\hline Transcription factors & NFKB1 & Nuclear factor-кB1 & 74 \\
\hline Angiogenesis & VEGF & Vascular endothelial growth factor & 75 \\
\hline \multirow[t]{8}{*}{ Others } & LSP1 & Lymphocyte-specific protein 1 & 66 \\
\hline & PMS1 and PMS2 & Postmeiotic segregation increased $1 / 2$ & 76 \\
\hline & RUVBL1 & RuvB-like 1 & 43 \\
\hline & CASP5 & Caspase-5, apoptosis-related cysteine peptidase & 43 \\
\hline & NXPH2 & Neurexophilin 2 & 77 \\
\hline & TERT & Telomerase reverse transcriptase & 67 \\
\hline & MSL1 & Male-specific lethal-1 homolog & 68 \\
\hline & PRPF31 & PRP31 pre-mRNA processing factor 31 homolog & 68 \\
\hline
\end{tabular}

The susceptibility genes verified to be expressed in EOC. They are associated with increased risk of EOC.

tumors, and usually have mutations in the TP53 tumor suppressor gene $(58,59)$. They also have germline or somatic mutations in BRCA1 or BRCA2, or promoter methylation of BRCA1 with loss of expression or function (60). MACs most probably arise via an adenoma-borderline tumor-carcinoma sequence with mutations of KRAS (59). Mutations of CTNNB1 [catenin (cadherin-associated protein), $\beta 1,88 \mathrm{kDa}$ ], the gene encoding $\beta$-catenin, are common in EAC but rare in SAC, MAC and CCC (59). Mutations of PIK3CA (phosphoinositide3 -kinase, catalytic, $\alpha$ polypeptide), which encodes the catalytic subunit of PI3K (phosphoinositide 3-kinase), are observed most frequently in CCC (59). Recent genetic studies implicate ARID1A [AT rich interactive domain 1A (SWI-like)] as a tumor-suppressor gene frequently disrupted in CCC and EAC $(61,62)$. Since ARID1A mutation can be seen in the preneoplastic lesions (atypical endometriosis), this is an early event in the transformation of endometriosis into cancer. This model postulates the existence of additional mutations or dysfunction that establish carcinogenesis after acquisition of ARID1A mutations and the onset of disease phenotype. These studies not only represent the remarkable genetic research achievements in the pathogenesis of EAOC, but also propose a direction of research in the future.

Mouse models of EOC using transgenic mice or viral vectors have been developed and show promise to more accurately recapitulate human disease, accelerate ovarian tumor research and predict clinical efficacy. Specific genetic defects are likely to be present in other histological types of EOC. Models of the serous histological subtype are based on Tg-MISIIR-TAg mice expressing the SV40 transforming region under control of the Müllerian inhibitory substance type II receptor (MISIIR) gene promoter (63). The oncogenic KRAS and loss of function of PTEN represents the mouse model that spontaneously develops endometrioid type of tumors (64). Promising results in animal models point to a future clinical application of this therapeutic strategy.

Loss of TP53 or BRCA function and inability to repair double-strand DNA repair breaks lead to chromosomal instability and apoptosis. Inhibition of the DNA repair is an attractive therapeutic target for drugs (58). For example, inhibition of the DNA repair enzyme poly(ADP-ribose) polymerase 1 (PARP-1) is a recently developed strategy for cancer therapy (65). PARP-1 inhibition can enhance the accumulation of DNA strand breaks and promote genomic instability and then apoptosis. PARP-1 inhibition is toxic to cells with mutations in the ovarian cancer susceptibility genes BRCA1 or BRCA2. Therefore, these inhibitors are an exciting new class of targeted therapy for treating patients with homologous recombination (HR; BRCA1 and BRCA2) repair-defective tumors (65).

Finally, there are many studies describing a candidate SNP approach to search for common, germline genetic variants associated with EOC risk (66). Variants in genes expressed as a consequence of interactions between EOC cells and the host 
micro-environment could contribute to cancer susceptibility (67). Recently, the majority of EOC candidate gene studies and genome-wide linkage analyses have been performed on populations of Japanese descent (Table II). Genetic variations of the following genes are considered to be contributors to the development of EOC.

For EOC, the known highly penetrant susceptibility genes (BRCA1 and BRCA2) are probably responsible for only $40 \%$ of the excess familial EOC risks, suggesting that other susceptibility genes of lower penetrance exist (66). Furthermore, there are several common moderate-low-penetrance susceptibility genes for EOC, including hormone receptors, cell cycle regulators, cytokine modulators and others.

Analysis of HOXD1 (homeobox D1), MYC, TIPARP [TCDD-inducible poly(ADP-ribose) polymerase] and SKAP1 (src kinase associated phosphoprotein 1) at these loci and of BNC2 (basonuclin 2) at 9p22, HEXIM1 (hexamethylene bisacetamide inducible 1) rs1053578, a common allele of STAG3 (stromal antigen 3) and HEXIM1 rs1053578 supports a functional role for these genes in EOC development and increased risk (68-70). SNPs in MFSD7 (major facilitator superfamily domain containing 7), BTN3A3 (butyrophilin, subfamily 3 , member A3), ZNF200 (zinc finger protein 200), and PTPRS (protein tyrosine phosphatase, receptor type, $\mathrm{S}$ ) were inversely associated with risk (68).

\section{Future research}

This review summarizes and evaluates the current evidence for the genetics and epidemiology of EOC, emphasizing the focus on CCC pathogenesis. Epithelial cancers are extremely common ovarian cancers. Recent studies brought about a new paradigm for the pathogenesis and origin of EOC (2). EOC is composed of a heterogeneous group of tumors, type I and type II tumors. Type I tumors (low-grade SAC, MAC, EAC and $\mathrm{CCC}$ ) are a distinct entity that is unrelated to type II tumors (high-grade SAC). Type I tumors, CCCs and EACs, are frequently associated with endometriosis. Type II tumors may develop outside the ovary (2). Kurman and Shih have raised speculation that 'true ovarian tumors' are non-epithelial neoplasms analogous to testicular tumors (2).

Recent epidemiologic studies identified a strong association between endometriosis and increased risk of developing CCC in Japanese women. CCC occurs more frequently in Japan (27.6\%) than in Western countries (about 10\%), possibly with fewer SAC cases (40.7\%). This may reflect a proportional increase in CCC or decrease in SAC. Several molecular genetic changes in CCC have been reported and some part of the molecular pathogenesis was unveiled. ARID1A mutation is an early event in the transformation of endometriosis into EOC (61). The late event of molecular genetic changes associated with $\mathrm{CCC}$ remain to be identified.

Finally, the study of EOC includes four major areas of interest: the study of the spread in mass population by careful data collection, the epidemiological study of causal risk factors, the study of carcinogenesis by modern genetic techniques, and an experimental study to verify the prior identified hypothesis. It has been reported that genetic factors related to hormone receptors, cell cycle regulators, cytokines, DNA repair or detoxification pathways may confer different degrees of susceptibility or ethnicity to endometriosis-related ovarian carcinogenesis.

At present, however, the insight gained from examining individual factors, including environmental factors, is limited. The environmental factors contain not only age, parity, diet, family history and ethnicity, but also tumormicroenvironmental factors such as persistent oxidative stress and inflammatory states. In women with endometriosis, retrograde menstruation or ovarian hemorrhage carries highly pro-oxidant factors, such as iron, into the peritoneal cavity or ovarian endometrioma. The repeated events of hemorrhage in endometriosis can contribute to carcinogenesis and progression via oxidative stress-induced gene mutation and aberrant expression of stress signaling pathways. A persistent oxidative stress-induced accumulation of damaged DNA and the consequent chromosomal instability may be a vital molecular mechanism during the process of multistep iron-induced CCC carcinogenesis. Some studies suggest a gene-oxidative stress interaction for CCC risk (56). We consider the roles of oxidative stress, DNA repair, chromatin remodeling, somatic mutations and microenvironmental changes in developing CCC.

The following objectives will be achieved by means of several research approaches. The common epidemiological characteristics of endometriosis and CCC need to be clarified. The precise molecular mechanisms involved in the pathogenesis of endometriosis and CCC need to be identified. Thus, common, shared and disparate genetic and epigenetic risk factors and their interactions need to be clarified. Future research should focus on the pathogenesis of oxidative stress-induced CCC carcinogenesis. Further clarification of the significance of specific genetic and environmental factors may lead to progress in the primary prevention and chemoprevention of CCC.

In conclusion, Japan has the highest incidence rates of $\mathrm{CCC}$ in the world and endometriosis in Japanese women may be significantly associated with CCC. Future genome-wide association studies should identify multiple EOC risk with regard to the key SNPs in the inflammation and oxidative stress-associated candidate regions and the risk of CCC in a Japanese population.

\section{Acknowledgements}

This review was supported by KAKENHI [Japan Society for the Promotion of Science (JSPS) Grant-in-Aid]. We thank all the study participants for their time and efforts. We thank Mr. Mikiko Kita for editorial assistance.

\section{References}

1. Kurman RJ and Shih IeM: The origin and pathogenesis of epithelial ovarian cancer: a proposed unifying theory. Am J Surg Pathol 34: 433-443, 2010.

2. Kurman RJ and Shih IeM: Molecular pathogenesis and extraovarian origin of epithelial ovarian cancer-shifting the paradigm. Hum Pathol 42: 918-931, 2011.

3. Goodman MT and Shvetsov YB: Incidence of ovarian, peritoneal, and fallopian tube carcinomas in the United States, 1995-2004. Cancer Epidemiol Biomarkers Prev 18: 132-139, 2009.

4. Rosenblatt KA and Thomas DB: Reduced risk of ovarian cancer in women with a tubal ligation or hysterectomy. The World Health Organization Collaborative Study of Neoplasia and Steroid Contraceptives. Cancer Epidemiol Biomarkers Prev 5: 933-935, 1996. 
5. Seidman JD, Yemelyanova A, Zaino RJ and Kurman RJ: The fallopian tube-peritoneal junction: a potential site of carcinogenesis. Int J Gynecol Pathol 30: 4-11, 2011.

6. Carlson JW, Miron A, Jarboe EA, Parast MM, Hirsch MS Lee Y, Muto MG, Kindelberger D and Crum CP: Serous tuba intraepithelial carcinoma: its potential role in primary peritoneal serous carcinoma and serous cancer prevention. J Clin Oncol 26: 4160-4165, 2008.

7. Massuger L, Roelofsen T, Ham M and Bulten J: The origin of serous ovarian cancer may be found in the uterus: a novel hypothesis. Med Hypotheses 74: 859-861, 2010.

8. McGuire V, Jesser CA and Whittemore AS: Survival among U.S women with invasive epithelial ovarian cancer. Gynecol Oncol 84: 399-403, 2002.

9. Scully RE: Pathology of ovarian cancer precursors. J Cell Biochem (Suppl) 23: 208-218, 1995.

10. Roett MA and Evans P: Ovarian cancer: an overview. Am Fam Physician 80: 609-616, 2009.

11. Jemal A, Tiwari RC, Murray T, Ghafoor A, Samuels A, Ward E, Feuer EJ and Thun MJ; American Cancer Society: Cancer statistics, 2004. CA Cancer J Clin 54: 8-29, 2004.

12. Aris A: Endometriosis-associated ovarian cancer: a ten-year cohort study of women living in the Estrie Region of Quebec, Canada. J Ovarian Res 3: 2, 2010.

13. Rice LW: Hormone prevention strategies for breast, endometrial and ovarian cancers. Gynecol Oncol 118: 202-207, 2010.

14. Winter WE III, Maxwell GL, Tian C, Carlson JW, Ozols RF, Rose PG, Markman M, Armstrong DK, Muggia F and McGuire WP: Prognostic factors for stage III epithelial ovarian cancer: a Gynecologic Oncology Group Study. J Clin Oncol 25: 3621-3627, 2007

15. Pisani P, Parkin DM, Bray F and Ferlay J: Estimates of the worldwide mortality from 25 cancers in 1990. Int J Cancer 83: 18-29, 1999.

16. Sugiyama T, Kamura T, Kigawa J, Terakawa N, Kikuchi Y, Kita T, Suzuki M, Sato I and Taguchi K: Clinical characteristics of clear cell carcinoma of the ovary: a distinct histologic type with poor prognosis and resistance to platinum-based chemotherapy. Cancer 88: 2584-2589, 2000.

17. Chan JK, Zhang M, Hu JM, Shin JY, Osann K and Kapp DS Racial disparities in surgical treatment and survival of epithelial ovarian cancer in United States. J Surg Oncol 97: 103-107, 2008

18. Ness RB, Grisso JA, Cottreau C, Klapper J, Vergona R, Wheeler JE, Morgan M and Schlesselman JJ: Factors related to inflammation of the ovarian epithelium and risk of ovarian cancer. Epidemiology 11: 111-117, 2000.

19. Tung KH, Goodman MT, Wu AH, McDuffie K, Wilkens LR, Kolonel LN, Nomura AM, Terada KY, Carney ME and Sobin LH: Reproductive factors and epithelial ovarian cancer risk by histologic type: a multiethnic case-control study. Am J Epidemiol 158: 629-638, 2003

20. Auersperg N, Wong AS, Choi KC, Kang SK and Leung PC: Ovarian surface epithelium: biology, endocrinology, and pathology. Endocr Rev 22: 255-288, 2001.

21. Crum CP, Drapkin R, Miron A, Ince TA, Muto M, Kindelberger DW and Lee Y: The distal fallopian tube: a new model for pelvic serous carcinogenesis. Curr Opin Obstet Gynecol 19: 3-9, 2007.

22. Varella-Garcia M: Chromosomal and genomic changes in lung cancer. Cell Adh Migr 4: 100-106, 2010.

23. Ripperger T, Gadzicki D, Meindl A and Schlegelberger B: Breast cancer susceptibility: current knowledge and implications for genetic counselling. Eur J Hum Genet 17: 722-731, 2009.

24. McLemore MR, Miaskowski C, Aouizerat BE, Chen LM and Dodd MJ: Epidemiological and genetic factors associated with ovarian cancer. Cancer Nurs 32: 281-288, 2009.

25. Prentice RL, Thomson CA, Caan B, Hubbell FA, Anderson GL, Beresford SA, Pettinger M, Lane DS, Lessin L, Yasmeen S, Singh B, et al: Low-fat dietary pattern and cancer incidence in the Women's Health Initiative Dietary Modification Randomized Controlled Trial. J Natl Cancer Inst 99: 1534-1543, 2007.

26. Greiser CM, Greiser EM and Dören M: Menopausal hormone therapy and risk of ovarian cancer: systematic review and metaanalysis. Hum Reprod Update 13: 453-463, 2007.

27. Mørch LS, Løkkegaard E, Andreasen AH, Krüger-Kjaer S and Lidegaard O: Hormone therapy and ovarian cancer. JAMA 302 298-305, 2009

28. Purdie DM, Bain CJ, Siskind V, Russell P, Hacker NF, Ward BG, Quinn MA and Green AC: Hormone replacement therapy and risk of epithelial ovarian cancer. Br J Cancer 81: 559-563, 1999.
29. Vang R, Whitaker BP, Farhood AI, Silva EG, Ro JY and Deavers MT: Immunohistochemical analysis of clear cell carcinoma of the gynecologic tract. Int J Gynecol Pathol 20: 252-259, 2001

30. Fujimura M, Hidaka T, Kataoka K, Yamakawa Y, Akada S, Teranishi A and Saito S: Absence of estrogen receptor-alpha expression in human ovarian clear cell adenocarcinoma compared with ovarian serous, endometrioid, and mucinous adenocarcinoma. Am J Surg Pathol 25: 667-672, 2001.

31. Clendenen TV, Lundin E, Zeleniuch-Jacquotte A, Koenig KL, Berrino F, Lukanova A, Lokshin AE, Idahl A, Ohlson N, Hallmans G, et al: Circulating inflammation markers and risk of epithelial ovarian cancer. Cancer Epidemiol Biomarkers Prev 20: 799-810, 2011.

32. Kobayashi H, Sumimoto K, Moniwa N, Imai M, Takakura K, Kuromaki T, Morioka E, Arisawa K and Terao T: Risk of developing ovarian cancer among women with ovarian endometrioma: a cohort study in Shizuoka, Japan. Int J Gynecol Cancer 17: 37-43, 2007.

33. Kobayashi H: Ovarian cancer in endometriosis: epidemiology, natural history, and clinical diagnosis. Int J Clin Oncol 14: 378-382, 2009.

34. Mandai M, Yamaguchi K, Matsumura N, Baba T and Konishi I: Ovarian cancer in endometriosis: molecular biology, pathology, and clinical management. Int J Clin Oncol 14: 383-391, 2009

35. Yamaguchi K, Mandai M, Toyokuni S, Hamanishi J, Higuchi T, Takakura K and Fujii S: Contents of endometriotic cysts, especially the high concentration of free iron, are a possible cause of carcinogenesis in the cysts through the iron-induced persistent oxidative stress. Clin Cancer Res 14: 32-40, 2008.

36. Kobayashi H, Kajihara H, Yamada Y, Tanase Y, Kanayama S, Furukawa N, Noguchi T, Haruta S, Yoshida S, Naruse K, Sado T and $\mathrm{Oi} \mathrm{H}$ : Risk of carcinoma in women with ovarian endometrioma. Front Biosci (Elite Ed) 3: 529-539, 2011.

37. Toyokuni S: Mysterious link between iron overload and CDKN2A/2B. J Clin Biochem Nutr 48: 46-49, 2011.

38. Toyokuni S: Role of iron in carcinogenesis: cancer as a ferrotoxic disease. Cancer Sci 100: 9-16, 2009.

39. Weiss NS and Peterson AS: Racial variation in the incidence of ovarian cancer in the United States. Am J Epidemiol 107: 91-95, 1978.

40. Singh P, Arunachalam I, Singh P, Tan BY, Tock EP and Ratnam SS: Ovarian cancer in Oriental women from Singapore: disease pattern and survival. Int Surg 75: 115-122, 1990

41. Chan JK, Teoh D, Hu JM, Shin JY, Osann K and Kapp DS: Do clear cell ovarian carcinomas have poorer prognosis compared to other epithelial cell types? A study of 1411 clear cell ovarian cancers. Gynecol Oncol 109: 370-376, 2008.

42. Köbel M, Kalloger SE, Huntsman DG, Santos JL, Swenerton KD, Seidman JD and Gilks CB: Differences in tumor type in low-stage versus high-stage ovarian carcinomas. Int J Gynecol Pathol 29: 203-211, 2010.

43. Notaridou M, Quaye L, Dafou D, Jones C, Song H, Høgdall E, Kjaer SK, Christensen L, Høgdall C and Blaakaer J: Common alleles in candidate susceptibility genes associated with risk and development of epithelial ovarian cancer. Int J Cancer 128: 2063-2074, 2011

44. Mink PJ, Sherman ME and Devesa SS: Incidence patterns of invasive and borderline ovarian tumors among white women and black women in the United States. Results from the SEER Program, 1978-1998. Cancer 95: 2380-2389, 2002.

45. Orezzoli JP, Russell AH, Oliva E, Del Carmen MG, Eichhorn J and Fuller AF: Prognostic implication of endometriosis in clear cell carcinoma of the ovary. Gynecol Oncol 110: 336-344, 2008.

46. Ogawa S, Kaku T, Amada S, Kobayashi H, Hirakawa T, Ariyoshi K, Kamura T and Nakano H: Ovarian endometriosis associated with ovarian carcinoma: a clinicopathological and immunohistochemical study. Gynecol Oncol 77: 298-304, 2000.

47. Surprasert P, Khunamornpong S and Srisomboon J: Clinicopathological features and prognosis of Thai women with endometriosis-associated ovarian carcinoma. Asian Pac J Cancer Prev 7: 638-640, 2006.

48. Vercellini P, Parazzini F, Bolis G, Carinelli S, Dindelli M, Vendola N, Luchini L and Crosignani PG: Endometriosis and ovarian cancer. Am J Obstet Gynecol 169: 181-182, 1993.

49. Kawaguchi R, Tsuji Y, Haruta S, Kanayama S, Sakata M, Yamada Y, Fujita H, Saito H, Tsuneto K and Kobayashi H: Clinicopathologic features of ovarian cancer in patients with ovarian endometrioma. J Obstet Gynaecol Res 34: 872-877, 2008 . 
50. Jiang X, Morland SJ, Hitchcock A, Thomas EJ and Campbell IG: Allelotyping of endometriosis with adjacent ovarian carcinoma reveals evidence of a common lineage. Cancer Res 58: 1707-1712, 1998.

51. Barlow DH and Kennedy S: Endometriosis: new genetic approaches and therapy. Annu Rev Med 56: 345-356, 2005.

52. Berkley KJ, Rapkin AJ and Papka RE: The pains of endometriosis. Science 308: 1587-1589, 2005.

53. Jacoby VL, Fujimoto VY, Giudice LC, Kuppermann $M$ and Washington AE: Racial and ethnic disparities in benign gynecologic conditions and associated surgeries. Am J Obstet Gynecol 202: 514-521, 2010.

54. Brinton LA, Lamb EJ, Moghissi KS, Scoccia B, Althuis MD, Mabie JE and Westhoff CL: Ovarian cancer risk associated with varying causes of infertility. Fertil Steril 82: 405-414, 2004.

55. Zhao C, Wu LS and Barner R: Pathogenesis of ovarian clear cell adenofibroma, atypical proliferative (borderline) tumor, and carcinoma: clinicopathologic features of tumors with endometriosis or adenofibromatous components support two related pathways of tumor development. J Cancer 2: 94-106, 2011.

56. Kajihara H, Yamada Y, Kanayama S, Furukawa N, Noguchi $T$, Haruta S, Yoshida S, Sado T, Oi H and Kobayashi H: Clear cell carcinoma of the ovary: Potential pathogenic mechanisms (Review). Oncol Rep 23: 1193-1203, 2010.

57. Kobayashi H, Yamada Y, Kanayama S, Furukawa N, Noguchi T, Haruta S, Yoshida S, Sakata M, Sado T and Oi H: The role of hepatocyte nuclear factor-1beta in the pathogenesis of clear cell carcinoma of the ovary. Int J Gynecol Cancer 19: 471-479, 2009.

58. Gilks CB: Molecular abnormalities in ovarian cancer subtypes other than high-grade serous carcinoma. J Oncol 2010: 740968, 2010.

59. Cho KR: Ovarian cancer update: lessons from morphology, molecules, and mice. Arch Pathol Lab Med 133: 1775-1781, 2009.

60. Press JZ, De Luca A, Boyd N, Young S, Troussard A, Ridge Y, Kaurah P, Kalloger SE, Blood KA, Smith M, et al: Ovarian carcinomas with genetic and epigenetic BRCA1 loss have distinct molecular abnormalities. BMC Cancer 8: 17, 2008.

61. Wiegand KC, Shah SP, Al-Agha OM, Zhao Y, Tse K, Zeng T, Senz J, McConechy MK, Anglesio MS, Kalloger SE, et al: ARID1A mutations in endometriosis-associated ovarian carcinomas. N Engl J Med 363: 1532-1543, 2010.

62. Jones S, Wang TL, Shih IeM, Mao TL, Nakayama K, Roden R, Glas R, Slamon D, Diaz LA Jr, Vogelstein B, Kinzler KW, Velculescu VE and Papadopoulos N: Frequent mutations of chromatin remodeling gene ARID1A in ovarian clear cell carcinoma. Science 330: 228-231, 2010

63. Nunez-Cruz S, Connolly DC and Scholler N: An orthotopic model of serous ovarian cancer in immunocompetent mice for in vivo tumor imaging and monitoring of tumor immune responses. $\mathrm{J}$ Vis Exp 45: 2146, 2010.

64. Dinulescu DM, Ince TA, Quade BJ, Shafer SA, Crowley D and Jacks T: Role of K-ras and Pten in the development of mouse models of endometriosis and endometrioid ovarian cancer. Nat Med 11: 63-70, 2005.

65. Wiltshire TD, Lovejoy CA, Wang T, Xia F, O'Connor MJ and Cortez D: Sensitivity to poly(ADP-ribose) polymerase (PARP) inhibition identifies ubiquitin-specific peptidase 11 (USP11) as a regulator of DNA double-strand break repair. J Biol Chem 285 : 14565-14571, 2010.
66. Quaye L, Tyrer J, Ramus SJ, Song H, Wozniak E, DiCioccio RA, McGuire V, Høgdall E, Høgdall C, Blaakaer J, et al: Association between common germline genetic variation in 94 candidate genes or regions and risks of invasive epithelial ovarian cancer. PLoS One 4: e5983, 2009.

67. Johnatty SE, Beesley J, Chen X, Macgregor S, Duffy DL, Spurdle AB, deFazio A, Gava N, Webb PM and Rossing M: Evaluation of candidate stromal epithelial cross-talk genes identifies association between risk of serous ovarian cancer and TERT, a cancer susceptibility 'hot-spot'. PLoS Genet 6: e1001016, 2010.

68. Peedicayil A, Vierkant RA, Hartmann LC, Fridley BL, Fredericksen ZS, White KL, Elliott EA, Phelan CM, Tsai YY, Berchuck A, Iversen ES Jr, et al: Risk of ovarian cancer and inherited variants in relapse-associated genes. PLoS One 5: e8884, 2010.

69. Goode EL, Chenevix-Trench G, Song H, Ramus SJ, Notaridou M, Lawrenson K, Widschwendter M, Vierkant RA, Larson MC and Kjaer SK: A genome-wide association study identifies susceptibility loci for ovarian cancer at $2 \mathrm{q} 31$ and $8 \mathrm{q} 24$. Nat Genet 42 : 874-879, 2010.

70. Ludwig AH, Murawska M, Panek G, Timorek A and Kupryjanczyk J: Androgen, progesterone, and FSH receptor polymorphisms in ovarian cancer risk and outcome. Endocr Relat Cancer 16: 1005-1016, 2009.

71. Beesley J, Jordan SJ, Spurdle AB, Song H, Ramus SJ, Kjaer SK, Hogdall E, DiCioccio RA, McGuire V and Whittemore AS: Association between single-nucleotide polymorphisms in hormone metabolism and DNA repair genes and epithelial ovarian cancer: results from two Australian studies and an additional validation set. Cancer Epidemiol Biomarkers Prev 16: 2557-2565, 2007.

72. Bolton KL, Tyrer J, Song H, Ramus SJ, Notaridou M, Jones C, Sher T, Gentry-Maharaj A, Wozniak E and Tsai YY: Common variants at $19 \mathrm{p} 13$ are associated with susceptibility to ovarian cancer. Nat Genet 42: 880-884, 2010.

73. Palmieri RT, Wilson MA, Iversen ES, Clyde MA, Calingaert B, Moorman PG, Poole C, Anderson AR, Anderson S and Anton-Culver H: Polymorphism in the IL18 gene and epithelial ovarian cancer in non-Hispanic white women. Cancer Epidemiol Biomarkers Prev 17: 3567-3572, 2008.

74. Fan Y, Yu W, Ye P, Wang H, Wang Z, Meng Q, Duan Y, Liang X and An W: NFKB1 insertion/deletion promoter polymorphism increases the risk of advanced ovarian cancer in a Chinese population. DNA Cell Biol 30: 241-245, 2011

75. Li Y, Wang Y, Kang S, Wang N, Zhou RM, Duan YN, Sun DL, Qin JJ, Zhao W and Zhao L: Association of vascular endothelial growth factor gene polymorphisms with susceptibility to epithelial ovarian cancer. Int J Gynecol Cancer 20: 717-723, 2010.

76. Mann A, Hogdall E, Ramus SJ, DiCioccio RA, Hogdall C, Quaye L, McGuire V, Whittemore AS, Shah M, Greenberg D, et al: Mismatch repair gene polymorphisms and survival in invasive ovarian cancer patients. Eur J Cancer 44: 2259-2265, 2008.

77. Song H, Ramus SJ, Kjaer SK, DiCioccio RA, ChenevixTrench G, Pearce CL, Hogdall E, Whittemore AS, McGuire V and Hogdall C: Association between invasive ovarian cancer susceptibility and 11 best candidate SNPs from breast cancer genome-wide association study. Hum Mol Genet 18: 2297-2304, 2009. 